\title{
Adherence to the American Heart Association Recommended Healthy Diet and Prevalence of Metabolic Syndrome in Male Japanese Workers
}

\author{
Masao Kanauchi ${ }^{1 *}$, Kimiko Kanauchi ${ }^{2}$ \\ ${ }^{1}$ Department of Health and Nutrition, Faculty of Health Science, Kio University 4-2-2 Umami-naka, Koryo-cho, \\ Kitakatsuragi District, Nara, Japan \\ ${ }^{2}$ Department of Internal Medicine, Narahigashi Hospital, Tenri, Nara, Japan 470 Nakanosho-cho, Tenri, Nara 632- \\ 0001, Japan
}

Received: September 5, 2018; Accepted: September 28, 2018; Published: October 4, 2018

*Corresponding author: Masao Kanauchi, Department of Health and Nutrition, Faculty of Health Science, Kio University 4-2-2 Umami-naka, Koryo-cho, Kitakatsuragi District, Nara, Japan. E-mail: m.kanauchi@kio.ac.jp

\begin{abstract}
Objective: The American Heart Association-recommended healthy diet (AHA-HD) is one of 7 ideal cardiovascular health metrics. Few studies have focused on use of the AHA-HD in Asian subjects. This study reports on the prevalence of adherence to the AHA-HD and examines the association between adherence to this diet and the risk for metabolic syndrome in male Japanese workers.
\end{abstract}

Design: A cross-sectional study provided data from 508 male workers, aged 25 to 63 years. Participants completed a 58-item brieftype, self-administered dietary history questionnaire. The AHA-HD score include 8 components (fruits and vegetables, fish, whole grains, salt, sugar-sweetened beverages, processed meat, legumes, and saturated fatty acids).

Results: The AHA-HD score of all subjects ranged from 0 to 7 ; no participant had a full score for all 8 components. The prevalence of metabolic syndrome was significantly lower in subjects with high adherence to AHA-HD (met $\geq 4$ components) than in those with low adherence $(21.8 \%$ vs. $30.1 \%$, respectively). High adherence to the AHA-HD was significantly associated with a low prevalence of metabolic syndrome $(\mathrm{OR}, 0.64 ; 95 \% \mathrm{CI}, 0.42-0.97 ; \mathrm{p}=0.038)$ after adjustment for age, total energy intake, smoking habit, and alcohol drinking.

Conclusions: Improving adherence to the AHA-HD recommendations may be an effective approach to promoting better health. Achievement of eating behaviors that meet $\geq 4$ components of the AHA-HD may lead to a reduction in the risk for metabolic syndrome.

Key words: American Heart Association-recommended healthy diet; Japan; metabolic syndrome; workers;

\section{Introduction}

Diet quality has emerged as an alternative to the traditional approach of using single nutrients or food groups to examine associations between diet and health, and several dietary quality scores have been proposed to assess healthy eating patterns [1, 2]. In 2010, the American Heart Association (AHA) published its strategic impact goal recommendations [3]. These recommendations proposed new dietary guidelines to reduce cardiovascular disease mortality by $20 \%$ and improve cardiovascular health by $20 \%$ by the year 2020 and beyond [3]. The committee defined a construct of ideal cardiovascular health, including 3 physiological risk factors and 4 healthy behaviors [3]. The AHA-recommended healthy diet (AHA-HD) is one of 7 ideal cardiovascular health metrics. The primary dietary recommendations include 5 components (fruits and vegetables, fish, whole grains, salt, and sugar-sweetened beverages), and secondary dietary recommendations include 3 additional components (processed meat, legumes, and saturated fatty acids). These recommendations are simple to determine compared with other indices and can be used easily in healthcare settings, especially in Western countries [4]. Application of this score in other populations requires certain adaptations, because dietary cultures and eating habits differ between Western and Asian countries. Although a traditional Japanese diet has been recognized as healthy $[5,6]$, dietary habits in Japan are changing from the traditional Japanese diet to an American-style diet or other less-healthy eating habits [7]. Thus, it is important to assess the contemporary diet in Japan using an American-style or a Westernized diet pattern score. In addition, the prevalence of metabolic syndrome among the Japanese population has increased in recent decades, especially in adult male workers [8]. However, there is little evidence that evaluates the association of adherence to the AHA-HD with the risk for metabolic syndrome. In a previous short report, we examined 7 metrics of ideal cardiovascular health and the risk for metabolic syndrome in Japanese subjects, but a precise analysis of the AHA-HD components was not performed [9]. The aim of this study was to report on adherence to the AHA-HD in male Japanese workers and to estimate the association between metabolic syndrome and increased adherence to this diet. 


\section{Methods}

\section{Study participants}

A total of 535 male workers were invited to participate in this cross-sectional study. Participants were recruited from a selected workplace (an electronic products factory) in Nara Prefecture, Japan. Of these, 8 persons refused to participate. The remaining 527 eligible subjects were chosen to answer the diet history questionnaire. Of these, we excluded 12 subjects who did not complete the diet history questionnaire and 7 subjects who had implausibly low or high estimated caloric intake $(<800$ or $>4500$ kcal per day). Finally, a total of 508 participants (mean age, 45.6 \pm 7.1 years; range, 25 to 63 years) were included in the analysis. This study was performed in accordance with the Helsinki Declaration. Study protocols were approved by the Institutional Review Board of Kio University, and written informed consent was obtained from each participant.

\section{Dietary assessment}

The dietary assessment is described in detail elsewhere [10] Briefly, using the 58-item brief self-administered diet history questionnaire (BDHQ) [11], participants were asked to choose 7 possible answers to indicate how often they had consumed specific foods during the past month (never, $<1$ time per week, once per week, 2-3 times per week, 4-6 times per week, once per day, and $\geq 2$ times per day). Values for the intake of nutrients and energy were estimated based on the questionnaire and the corresponding food composition list in the Standard Tables of Food Composition in Japan [12].

\section{The American Heart Association healthy diet score (AHA-HDS)}

We modified the original AHA-HD recommendations [3] to reflect cultural and dietary habits of Japanese subjects. The AHA-HDS includes 5 primary components (fruits and vegetables $\geq 400$ g per day; fish intake $\geq 2$ times per week with at least one of oily fish; fiber/carbohydrate ratio $\geq 0.1$; salt intake $<8 \mathrm{~g}$ per day; and sugar-sweetened beverages $<2$ times per week), plus 3 secondary components (processed meats $<2$ times per week; legumes $\geq 4$ times per week; and saturated fatty acid intake $<7 \%$ of total energy intake). Because measuring the precise amount of whole grain intake is difficult with the BDHQ we used a fiber/ carbohydrate ratio instead. Recommendations state that when choosing a whole grain food, a rule of $>1 \mathrm{~g}$ of fiber per $10 \mathrm{~g}$ of carbohydrate should be used [13]. This recommendation comes from a report from the Harvard School of Public Health [13] and has been used in previous studies assessing the AHA-HD $[14,15]$. Another modified point was the cutoff for salt intake. Because it is difficult to achieve the original AHA-HD criteria (sodium $<2300$ mg per day) in Japan, we used a value from a report of the Dietary Reference Intake for Japanese 2015 [16], which recommends <8 $\mathrm{g}$ of salt per day for men as a dietary goal for preventing lifestylerelated diseases. The AHA-HD was calculated as a dichotomous score, awarding 1 point for meeting the ideal definitions or 0 points for not meeting them, resulting in scores ranging from 0 to
8. The adherence to the AHA-HD was classified as high (met 4-8 components) and low (met 0-3 components).

\section{Assessment of metabolic syndrome}

Metabolic syndrome was defined according to the criteria of the International Diabetes Federation in conjunction with the National Heart, Lung, and Blood Institute, American Heart Association, World Heart Federation, International Atherosclerosis Society, and International Association for the Study of Obesity [17]. Subjects were defined as having metabolic syndrome if they had 3 or more of the following 5 components: waist circumference $\geq 85 \mathrm{~cm}$ [18]; high blood pressure ( $\geq 130 \mathrm{mmHg}$ systolic, $\geq 85 \mathrm{mmHg}$ diastolic, or current use of antihypertensive medicine); a high serum triglyceride level ( $\geq 150 \mathrm{mg} / \mathrm{dL}$ or specific treatment for this lipid abnormality); low high-density lipoprotein (HDL) cholesterol ( $<40 \mathrm{mg} / \mathrm{dL}$ ); and high fasting glucose level $(\geq 100 \mathrm{mg} / \mathrm{dL})$.

\section{other variables}

Body mass index (BMI) was calculated as weight in kilograms divided by the square of height in meters, and overweight/ obesity was defined as BMI $\geq 25 \mathrm{~kg} / \mathrm{m} 2$. Smoking status (yes, no) and physical activity (persons who exercised at least 2 days a week, 30 minutes or more each time, were defined as active) was assessed using a self-reported questionnaire. In addition, alcohol consumption (for men) was assessed using the BDHQ and was classified as low ( $<10 \mathrm{~g}$ of ethanol per day), moderate (10 to $30 \mathrm{~g}$ per day), and high ( $>30$ g per day).

\section{Statistical analyses}

Categorical variables were described as percentages and were compared by the chi-square test. Continuous variables between 2 groups were compared using Student's t-test. A binary logistic regression analysis was conducted to examine the association between adherence to the AHA-HD and the risk for metabolic syndrome. Three models were used. Model 1 was adjusted for age (years). Model 2 was adjusted for age (years), total energy intake (kcal/day), smoking (yes, no), and alcohol drinking (low, moderate, high). Model 3 was adjusted for model 2 components plus being physically active (yes, no). All statistical analyses were performed using SPSS version 21.0 (IBM Corp, Armonk, NY). P values $<0.05$ were considered statistically significant.

\section{Results}

\section{Characteristics of subjects with or without metabolic syndrome}

Characteristics and nutrient intakes of subjects with or without metabolic syndrome are shown in (Table 1). As expected, body weight, BMI, and waist circumference were significantly higher in participants with metabolic syndrome than in those without metabolic syndrome. Total energy intake and the proportion of energy intake from protein and carbohydrate were comparable between groups. Unexpectedly, the proportion of energy intake from fat was significantly lower in subjects with 
Table 1: Characteristics of subjects with or without metabolic syndrome

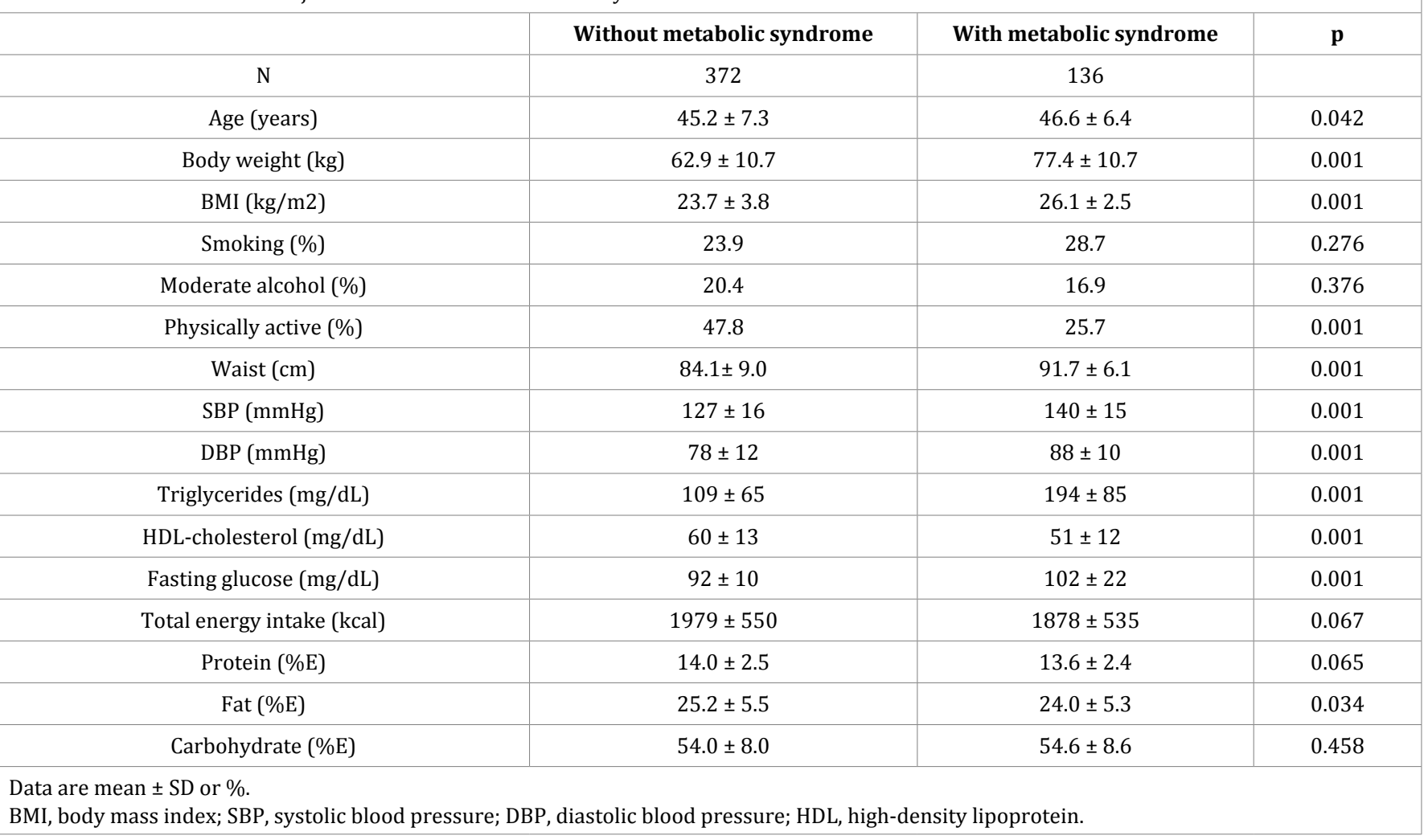

metabolic syndrome than those without. Among lifestyle factors, the proportion of subjects who were physically active was significantly higher in those without metabolic syndrome than those with.

\section{Distributions of AHA-HDS}

The AHA-HDS of all subjects ranged from 0 to 7; no participant had a full score for all 8 components. A high adherence rate (met $\geq 4$ components) was seen in $32.4 \%$ of subjects with metabolic syndrome and $42.5 \%$ of subjects without metabolic syndrome $(\mathrm{p}=0.039)$ (Table 2). The total AHA-HDS tended to be lower in subjects with metabolic syndrome than in subjects without metabolic syndrome, but differences were not significant (3.01 \pm 1.34 vs. $3.21 \pm 1.43$, respectively; $\mathrm{p}=0.150$ ).

Table 2: Distribution of AHA-HDS scores

\begin{tabular}{|c|c|c|c|}
\hline Number of Components & $\begin{array}{l}\text { All subjects } \\
\qquad(n=508)\end{array}$ & $\begin{array}{l}\text { Without metabolic syndrome } \\
\qquad(n=372)\end{array}$ & $\begin{array}{l}\text { With metabolic syndrome } \\
\qquad(n=136)\end{array}$ \\
\hline 0 & $11(2.2 \%)$ & $10(2.7)$ & $1(0.7)$ \\
\hline 1 & $51(10.0)$ & $32(8.6)$ & $19(14.0)$ \\
\hline 2 & 101 (19.9) & $75(20.2)$ & $26(19.1)$ \\
\hline 3 & $143(28.1)$ & $97(26.1)$ & $46(33.8)$ \\
\hline 4 & $117(23.6)$ & $91(24.5)$ & $26(19.1)$ \\
\hline 5 & $62(12.2)$ & $49(13.2)$ & $13(9.6)$ \\
\hline 6 & $18(3.5)$ & $14(3.8)$ & $4(2.9)$ \\
\hline 7 & $5(1.0)$ & $4(1.1)$ & $1(0.7)$ \\
\hline 8 & $0(0)$ & $0(0)$ & $0(0)$ \\
\hline
\end{tabular}




\section{Percentage of subjects meeting each component of the AHA-HD}

With respect to the individual components of the AHAHD recommendations, subjects were most likely to meet the ideal recommendations for saturated fatty acids, processed meat intake, and sugar-sweetened beverages, followed by fish intake and fruit and vegetable intake. Salt intake was least like to meet ideal guidelines, followed by fiber/carbohydrate ratio (representing high intakes of whole grain) (Table 3). Contrary to expectations, only sugar-sweetened beverage components were significantly lower in subjects with metabolic syndrome than in those without metabolic syndrome. Other components did not differ significantly between subjects with and without metabolic syndrome.

Table 3: Percentage of subjects meeting the different AHA-HD recommendations

\begin{tabular}{|c|c|c|c|c|}
\hline & All subjects & Without metabolic syndrome & With metabolic syndrome & $\mathbf{p}^{\mathbf{a}}$ \\
\hline Fruits and vegetables $\geq 400 \mathrm{~g} /$ day & 46.3 & 47.3 & 43.4 & 0.433 \\
\hline Fish $\geq 2$ times/week* & 47.2 & 47.3 & 47.1 & 0.960 \\
\hline Fiber/carbohydrate $\geq 0.1$ & 10.8 & 12.1 & 7.4 & 0.128 \\
\hline Salt $<8 \mathrm{~g} /$ day & 0.8 & 0.5 & 1.5 & 0.293 \\
\hline Processed meat $<2$ times/week & 60.2 & 59.4 & 62.5 & 0.529 \\
\hline Legumes $\geq 4$ times/week & 35.0 & 36.8 & 30.1 & 0.163 \\
\hline Saturated fatty acid $<7 \%$ of total energy & 63.2 & 61.8 & 66.9 & 0.294 \\
\hline
\end{tabular}

AHA-HDS, American Heart Association-recommended healthy diet score.

${ }^{a} \mathrm{P}$ values indicate difference between subjects with and without metabolic syndrome.

${ }^{*}$ At least 1 oily fish.

\section{Adherence to the AHA-HD and metabolic syndrome components}

The prevalence of metabolic syndrome was significantly lower in subjects with high adherence to AHA-HD recommendations than in subjects with low adherence to AHA-HD recommendations.
Among each component of metabolic syndrome, the prevalence of abdominal obesity, high triglycerides, and low HDL cholesterol levels were significantly lower in subjects with high adherence to the AHA-HD than in subjects with low adherence to the AHA-HD (Table 4).

\begin{tabular}{|c|c|c|c|}
\hline Components & $\begin{array}{c}\text { Low adherence to AHA-HD (0-3 } \\
\text { components) }\end{array}$ & $\begin{array}{l}\text { High adherence to AHA-HD (4-8 } \\
\text { components) }\end{array}$ & $\mathbf{P}$ \\
\hline Metabolic syndrome & $30.1 \%$ & $21.8 \%$ & 0.039 \\
\hline Abdominal obesity & 35.9 & 25.3 & 0.013 \\
\hline High blood pressure & 54.2 & 55.0 & 0.877 \\
\hline High triglycerides & 38.2 & 29.2 & 0.036 \\
\hline Low HDL cholesterol & 9.5 & 2.5 & 0.002 \\
\hline High fasting glucose & 21.9 & 26.7 & 0.211 \\
\hline \multicolumn{4}{|l|}{ Data are expressed as \%. } \\
\hline AHA-HDS, American Heart A & ommended healthy diet score. & & \\
\hline
\end{tabular}

\section{High adherence to AHA-HD and risk reduction for metabolic syndrome}

High adherence to the AHA-HD was associated with a low prevalence of metabolic syndrome compared with low adherence (logistic regression analysis) (Table 5). After adjustment for age (Model 1) and for age, total energy intake, smoking habit, and alcohol drinking (Model 2), high adherence to AHA-HD was significantly associated with low prevalence of metabolic syndrome. However, this association was attenuated in the fully adjusted model, when physically active status was added as an adjustment factor (Model 3). 
Table 5: High adherence to AHA-HD and risk reduction for the metabolic syndrome by multivariable-adjusted logistic regression analysis

\begin{tabular}{|c|c|c|c|}
\hline Model & Odds ratio & $\mathbf{9 5 \%}$ CI & p \\
\hline Crude & 0.65 & $0.43-0.98$ & 0.040 \\
\hline Model 1 & 0.61 & $0.40-0.93$ & 0.020 \\
\hline Model 2 & 0.64 & $0.42-0.97$ & 0.038 \\
\hline Model 3 & 0.67 & $0.44-1.04$ & 0.075 \\
\hline
\end{tabular}

Model 1: adjusted for age (years).

Model 2: adjusted for age (years), total energy intake (kcal/day), smoking (yes, no), alcohol drinking (low, moderate, high).

Model 3: adjusted for model 2 components plus physically active (yes, no).

CI, confidence interval.

\section{Discussion}

To the best of our knowledge, this is the first study to report on the prevalence of adherence to the AHA-HD overall as well as each component of the AHA-HD in male Japanese workers. There are few studies in Asian countries that report the status of ideal cardiovascular health metrics [19-25]. However, there is no report that evaluates the association between healthy dietary patterns and metabolic syndrome. One strength of the present study is that we especially focused on adherence to the AHA-HD, along with individual dietary components. Notably, no subject in our study had a full score for the AHA-HDS (maximum 8 points) and only $1.0 \%$ of subjects had a score of 7 points. One of the reasons for this finding was that ideal salt intake was not likely to meet the definition used in our study population. It is difficult to achieve the original AHA-HD criteria (sodium $<2300 \mathrm{mg}$ per day) in Japan, due to the high consumption of miso soup, salty fish, and Japanese pickles, all of which contain high levels of sodium and are commonly eaten foods in Japan [26]. It was reported that the average salt intake in Japanese men is 11.0 g per day [27]. Considering that, in this study, we corrected the upward cutoff of salt intake ( $<8 \mathrm{~g}$ per day) according to the report of the Dietary Reference Intake for Japanese 2015 [16]. Nevertheless, only $0.8 \%$ of subjects met this cutoff value. Many components of the AHAHD resemble a traditional Japanese diet: high intakes of fish, vegetables, and legumes, and low intakes of processed meat and sugar-sweetened beverages. In other words, persons who adhere to the AHA-HD might consume a healthy Japanese diet. However, the Japanese diet contains high salt intakes that are not seen in the AHA-HD. Similarly, the prevalence of high fiber/carbohydrate ratio, which represents a high intake of whole grains, was low in our study population. Two reasons for this finding are that (1) white rice is preferred in Japan compared to brown rice or other whole grain foods, and (2) white bread is also generally eaten in Japan. Therefore, it is reasonable that low prevalence of whole grain intake was seen in our data.

Adherence to this optimal diet was low in persons with metabolic syndrome. Several studies reported the prevalence of metabolic syndrome among various working populations, and these populations are often considered to have unhealthy eating habits [28]. Our results suggest that a healthy diet should be encouraged, especially in a working population. In addition, we found that an achievement of eating behaviors of $\geq 4$ components of the AHA-HD was associated with a lower risk for metabolic syndrome.

This study has some limitations. First, among the AHA-HD components, we modified some cutoffs or assessment tools from the original AHA-HD definitions. For example, the cutoff of salt intake was amended to correct upwards ( $<8 \mathrm{~g}$ per day), as described in the Methods section. The BDHQ used in this study is not designed to evaluate the total amount of whole grains, and therefore, whole grain intake was transformed to the ratio of dietary fiber to carbohydrate ( $\geq 0.1$ in this study). This definition comes from a report from the Harvard School of Public Health [13]. The Harvard researchers evaluated 545 grain products to find the best way to identify a healthful whole grain food: food that met the 10:1 ratio that tends to match unprocessed wheat [13]. The assessment for fruits and vegetables was also modified from the original AHA-HD definition ( $\geq 4.5$ servings per day) to the surrogate definition ( $\geq 400$ g per day), because use of serving units for fruits and vegetables is not common in Japan. Second, the lack of a randomly selected sample could lead to selection bias. Additional investigations including subjects with a wider range of occupations are needed. Third, education level, family income of participants, job stress, and the presence of shift work might contribute to adherence to a healthy diet, but we did not have such information. Finally, as a cross-sectional study, the present results cannot prove a causal relationship between the AHA-HD adherence and the risk reduction for metabolic syndrome. In addition, the independent effect of high adherence to AHA-HD was attenuated by the fully adjusted model (by adding physical activity as a confounding factor). Although it is commonly said that both physical activity and healthy dietary habits may contribute to a lower risk for metabolic syndrome [29], it is controversial whether physical activity or a healthier diet is more closely related to risk reduction of metabolic syndrome.

Despite these limitations, the main strength of our study was that we collected detailed data on dietary intakes and we could apply all 8 components of the AHA-HD. In recent decades, Japan has been replacing the traditional Japanese diet with an American-style diet or with other less healthy eating habits. This study adds new information regarding the lack of ideal healthy dietary status in contemporary Japan, and in particular, male workers. 
In conclusion, the prevalence of metabolic syndrome was significantly lower in subjects with high adherence to AHAHD (met $\geq 4$ components) than in those with low adherence ( $21.8 \%$ vs. $30.1 \%$, respectively). High adherence to the AHA-HD was significantly associated with a low prevalence of metabolic syndrome (OR, 0.64; 95\% CI, 0.42-0.97; $\mathrm{p}=0.038$ ) after adjustment for age, total energy intake, smoking habit, and alcohol drinking. Improvement of adherence to the AHA-HD may be an effective approach to promoting better health and reducing the risk for metabolic syndrome.

\section{Declarations}

a. Conflicts of interest: There is no conflict of interest to disclose.

b. Ethical Approval: This study was approved by the Institutional Review Board of Kio University.

c. Clinical trial registration: This was an observational study. Written informed consent was obtained from each participant.

\section{Data availability statement}

The datasets analyzed during the current study are available from the corresponding author upon reasonable request.

\section{Authors' contributions}

Masao Kanauchi conceived the study design, carried out data collection, analyzed the data, and drafted the paper. Kimiko Kanauchi helped with data collection and drafting the paper. All authors read and approved the final version of the paper.

\section{Funding statement}

This work was supported by JSPS KAKENHI Grant Number 17 K09339.

\section{References}

1. Alkerwi A. Diet quality concept. Nutrition. 2014;30(6):613-618. doi: 10.1016/j.nut.2013.10.001

2. Kourlaba G, Panagiotakos DB. Dietary quality indices and human health: A review. Maturitas. 2009;62(1):1-8. doi: 10.1016/j. maturitas.2008.11.021

3. Lloyd-Jones DM, Hong Y, Labarthe D, Mozaffarian D, Appel LJ, Van Horn L, et al. Defining and setting national goals for cardiovascular health promotion and disease reduction: the American Heart Association's strategic Impact Goal through 2020 and beyond. Circulation 2010;121(4):586-613. doi: 10.1161/CIRCULATIONAHA.109.192703

4. Rehm CD, Penalvo JL, Afshin A, Mozaffarian D. Dietary intake among US adults, 1999-2012. JAMA, 2016;315(23):2542-2553. doi: 10.1001/ jama.2016.7491

5. Niu K, Momma H, Kobayashi Y, Guan L, Chujo M, Otomo A, et al. The traditional Japanese dietary pattern and longitudinal changes in cardiovascular disease risk factors in apparently healthy Japanese adults. Eur J Nutr. 2016;55(1):267-279. doi: 10.1007/s00394-0150844-y
6. Nakamura Y, Ueshima H, Okamura T, Kadowaki T, Hayakawa T, Kita Y, et al. A Japanese diet and 19-year mortality: national integrated project for prospective observation of non-communicable diseases and its trends in the aged, 1980. Br J Nutr. 2009; 101(11):1696-705. doi:10.1017/S0007114508111503

7. Ministry of Health, Labour, and Welfare of Japan. The National Health and Nutrition Survey in Japan 2013. Ministry of Health, Labour, and Welfare. 2015.

8. Watanabe K, Sakuraya A, Kawakami N, Imamura K, Ando E, Asai Y, et al. Work-related psychosocial factors and metabolic syndrome onset among workers: a systematic review and meta-analysis. Obese Rev. 2018. doi: 10.1111/obr.12725

9. Kanauchi M, Kanauchi K. Prevalence and distribution of ideal cardiovascular health metrics and association with metabolic syndrome in Japanese male workers. Int J Cardiol. 2016;221:428-429. doi: 10.1016/j.ijcard.2016.07.017

10. Kanauchi M, Kanauchi K. Diet quality and associated factors among eldercare workers in long-term care facilities. J Gerontol Geriatr Res. 2016;5:360. doi: 10.4172/2167-7182.1000360

11. Kobayashi S, Murakami K, Sasaki S, Okubo H, Hirota N, Notsu $A$, et al. Comparison of relative validity of food group intakes estimated by comprehensive and brief-type self-administered diet history questionnaires against $16 \mathrm{~d}$ dietary records in Japanese adults. Public Health Nutr, 2011;14(7):1200-1211. doi: 10.1017/ S1368980011000504

12. Council for Science and Technology. Standard Tables of Food Composition in Japan, 2015. Ministry of Education, Culture, Sports, Science and Technology, 2015.

13. Mozaffarian RS, Lee RM, Kennedy MA, Ludwig DS, Mozaffarian D, Gortmaker SL. Identifying whole grain foods: a comparison of different approaches for selecting more healthful whole grain products. Public Health Nutr. 2013;16(12):2255-2264. doi: 10.1017/ S1368980012005447

14. Thacker EL, Gillett SR, Wadley VG, Unverzagt FW, Judd SE, McClure LA, et al. The American Heart Association Life's Simple 7 and incident cognitive impairment: The Reasons for Geographic and Racial Differences in Stroke (REGARDS) study. J Am Heart Assoc 2014;3(3):e000635. doi: 10.1161/JAHA.113.000635

15. Brewer LC, Redmond N, Slusser JP, Scott CG, Chamberlain AM, Djousse L, et al. Stress and achievement of cardiovascular health metrics: The American Heart Association Life's Simple 7 in Blacks of the Jackson Heart Study. J Am Heart Assoc, 2018;7: e008855. doi: 10.1161/ JAHA.118.008855

16. Ministry of Health, Labour and Welfare. Dietary Reference Intake for Japanese 2015. 
17. Alberti KG, Eckel RH, Grundy SM, Zimmet PZ, Cleeman JI, Donato KA, et al. Harmonizing the metabolic syndrome: a joint interim statement of the International Diabetes Federation Task Force on Epidemiology and Prevention; National Heart, Lung, and Blood Institute; American Heart Association; World Heart Federation; International Atherosclerosis Society; and International Association for the Study of Obesity. Circulation. 2009;120(16):1640-1645.

18. Yamagishi $\mathrm{K}$, Iso $\mathrm{H}$. The criteria for metabolic syndrome and the national health screening and education system in Japan. Epidemiol Health. 2017;39:e2017003. doi: 10.4178/epih.e2017003

19. Wu S, Huang Z, Yang X, Zhou Y, Wang A, Chen L, et al. Prevalence of ideal cardiovascular health and its relationship with the 4-year cardiovascular events in a north Chinese industrial city. Circ Cardiovasc Qual Outcomes. 2012;5(4):487-495. doi: 10.1161/ CIRCOUTCOMES.111.963694

20. Kim JY, Ko Y-J, Rhee CW, Park B-J, Kim D-H, Bae J-M, et al. Cardiovascular health metrics and all-cause and cardiovascular disease mortality among middle-aged men in Korea. J Prev Med Public Health 2013;46(6):319-328. doi.org/10.3961/jpmph.2013.46.6.319

21. Zeng Q Dong SY, Song ZY, Zheng YS, Wu HY, Mao LN. Ideal cardiovascular health in Chinese urban population. Int J Cardiol. 2013;167(5):2311 2317. doi: 10.1016/j.ijcard.2012.06.022

22. Liu Y, Chi HJ, Cui LF, Yang XC, Wu YT, Huang Z, et al. The ideal cardiovascular health metrics associated inversely with mortality from all causes and from cardiovascular diseases among adults in a Northern Chinese industrial city. PLoS One. 2014;9: e89161. doi 10.1371/journal.pone.0089161
23. Chou LP, Tsai CC, Li CY, Hu SC. Prevalence of ideal cardiovascular health and its relationship with job strain: a cross sectional study in Taiwanese medical employees. BMJ Open. 2016;6:e010467. doi: 10.1136/bmjopen-2015-010467

24. Zhou L, Zhao L, Wu Y, Wu Y, Gao X, Li Y, et al. Ideal cardiovascular health metrics and its association with 20-year cardiovascular morbidity and mortality in a Chinese population. J Epidemiol Community Health. 2017;72(8):752-758. doi: 10.1136/jech-2017-210396

25. Chung JW, Wong BY, Yan VC, Chung LM, So HC, Chan A. Cardiovascular Health of Construction Workers in Hong Kong: A Cross-Sectional Study. Int J Environ Res Public Health. 2018;15(6):E1251. doi: 10.3390/ ijerph15061251

26. Yamori Y, Sagara M, Arai Y, Kobayashi H, Kishimoto K, Matsuno I, et al. Soy and fish as features of the Japanese diet and cardiovascular disease risks. PLoS One. 2017;12:e0176039. doi: 10.1371/journal. pone. 0176039

27. Ministry of Health, Labour, and Welfare of Japan. The National Health and Nutrition Survey in Japan 2015. Ministry of Health, Labour, and Welfare, 2017.

28. Huang JH, Li RH, Huang SL, et al. Lifestyle factors and metabolic syndrome among workers. Int J Environ Res Public Health, 2015;12(12):15967-15978. doi: 10.3390/ijerph121215035

29. Silva TR, Alves BC, Maturana MA, Spritzer PM. Healthier dietary pattern and lower risk of metabolic syndrome in physically active postmenopausal women. J Am Coll Nutr. 2013;32:287-295. doi: $10.1080 / 07315724.2013 .826111$ 